\title{
Validação da versão em português do Inventário de Depressão Maior
}

\author{
Validation of the Portuguese version of the Major Depression Inventory
}

Silvia Parcias', Barbie P. do Rosario², Thiago Sakae², Fernanda Monte, Adriana C. A. Guimarães³, André J. Xavier²

\section{Palavras-chave}

Depressão, validação, questionários, transtornos do humor, inventário.

\section{Keywords}

Depression, validation, questionnaires, mood disorders, inventory.

\section{RESUMO}

Objetivo: Validar, adaptar e aferir a fidedignidade do Inventário de Depressão Maior (Major Depression Inventory - MDI) para a língua portuguesa. Métodos: $\mathrm{O}$ questionário passou pelo processo de adaptação transcultural. Foi realizado um pré-teste para avaliar sua aplicabilidade. Para avaliação de reprodutibilidade, utilizou-se medida repetida com intervalo de 1 a 2 semanas e coeficiente de correlação intraclasse. O MDI e a escala de Hamilton foram aplicados em 30 pacientes com diagnóstico de depressão, que foram pareados com 90 controles aos quais foi aplicado o MDI. A curva ROC foi realizada com 120 pacientes e escore final do MDI. Para análise da validade interna, utilizou-se o alfa de Cronbach. Resultados: Sensibilidade e especificidade foram 0,86 e 0,75, respectivamente, com escores 16/17. O alfa de Cronbach para a escala total foi 0,91. O coeficiente de Pearson entre o total do MDI e o total da escala de depressão de Hamilton foi 0,56. A análise fatorial revelou dois fatores: o primeiro explicava 53,9\% da variação enquanto o segundo explicava somente 13,6\%. A confiabilidade teste-reteste foi excelente (com coeficiente de correlação intraclasse variando de 0,50 e 0,93 para itens individuais e 0,90 para o escore total). Conclusão: As propriedades psicométricas do MDI se mostraram adequadas para aplicação na população brasileira, entretanto outros estudos se fazem necessários.

\section{ABSTRACT}

Objective: To validate, adapt and assess the reliability for the Major Depression Inventory (MDI) to the Portuguese language. Methods: The questionnary underwent a cultural adaptation process. A pre-test was made to check on its aplicability. To analise the reprodutibility, repeted mesuare was used in two oportunities, 1 and 2 weeks later, using the Intraclasses Correlation Coeficient. The MDI and the Hamilton Depression Escale were applied in 30 pacients with depression and pared with 90 controls to whom the MDI was applied to. The ROC curve was performed with 120 pacients and the final MDI score. The internal consistency was evaluated by means of Cronbach's alpha. Results: Sensitivity and specificity were 0.86 and 0.75 , respectively, with scors 16/17. Cronbach alfa's for the total scale was 0.91. The Pearson's coeficient between MDI and Hamilton depression scale was 0.56. The factor analisis revealed 2 factors: the first explained $53.9 \%$ of variance while the second explained only 13.6\%. The test-retest reliability was excellent (with intraclass coeficient correlation between 0.50 and 0.93 for individuals itens and 0.90 for total scale). Conclusion: MDI's properties showed to be appropriate to apply in the Brasilian population, but further studies are necessary.

\footnotetext{
1 Universidade do Estado de Santa Catarina (Udesc), Centro de Ciências da Saúde e do Esporte, Florianópolis, SC; Universidade do Sul de Santa Catarina (Unisul), Faculdade de Medicina, Tubarão, SC.

2 Universidade do Sul de Santa Catarina (Unisul), Faculdade de Medicina, Tubarão, SC.

3 Universidade do Estado de Santa Catarina (Udesc), Centro de Ciências da Saúde e do Esporte, Florianópolis, SC.
} 


\section{INTRODUÇÃO}

A depressão configura-se como um estado alterado do humor envolvendo tristeza, perda da capacidade de sentir prazer e, ainda, alterações cognitivas, motoras e somáticas, sendo que tais fatores interferem no funcionamento social do indivíduo'.

A prevalência anual de depressão na população geral varia de $3 \%$ a $11 \%$, ocorrendo mais nas mulheres que nos homens. É motivo de $10 \%$ das consultas em atenção primária. Representa a quarta causa mais importante de incapacidade no mundo, e acredita-se que se torne a segunda causa mais importante em 2020².

O Manual Diagnóstico e Estatístico de Transtornos Mentais (DSM-IV) classifica como depressão maior a presença, por pelo menos duas semanas, de 5 ou mais dos 9 sintomas: estado de humor depressivo, diminuição do prazer, perda de peso, insônia ou hipersonia, agitação ou lentidão, perda de energia, sentimento de inutilidade ou culpa, diminuição da capacidade de concentração e pensamentos recorrentes de mortes, sendo que, desses 5, um deve ser humor depressivo ou diminuição do prazer³. Já o sistema de Classificação Internacional de Doenças (CID-10), que inclui além dos sintomas do DSM-IV o sintoma falta de autoconfiança, categoriza depressão em leve, moderada e grave $e^{4}$.

Embora não se disponham de parâmetros fisiológicos ou biológicos para avaliar as manifestações clínicas da depressão, as escalas de avaliação servem para medir e caracterizar o fenômeno, isto é, traduzem o fenômeno clínico em informações objetivas e quantitativas ${ }^{5}$. Ajudam na avaliação dos sintomas e na elaboração do próprio diagnóstico, além de auxiliarem o acompanhamento do paciente e o resultado dos tratamentos ${ }^{6}$. Assim, é de extrema importância e utilidade para aplicação clínica e científica buscar instrumentos que auxiliem a estabelecer um diagnóstico preciso e recomendar o melhor tratamento.

Escalas autoaplicáveis são bastante utilizadas na pesquisa clínica, tais como o Inventário de Depressão Beck e a Escala de Depressão Autoaplicável de Zung, desenvolvidas nos anos 1960. A Escala de Avaliação de Depressão de Hamilton (HAM-D), desenvolvida há mais de 40 anos, é a mais aceita e usada no âmbito mundial e se tornou o padrão-ouro para avaliação da gravidade, de modo que as escalas desenvolvidas posteriormente são comparadas a ela quanto à confiabilidade e à validade. A HAM-D enfatiza os sintomas somáticos, o que a torna particularmente sensível a mudanças vivenciadas por pacientes gravemente deprimidos, e contribui para a difusão de seu uso em ensaios clínicos com antidepressivos ${ }^{7,8}$.

Entretanto, muitas dessas escalas de medida de gravidade e frequência de sintomas depressivos foram desenvolvidas antes de 1980, prévias ao advento dos sistemas de diagnóstico baseados em evidência o DSM-III, DSM-IV ou CID-10, e não cobrem todos os sintomas incluídos no algoritmo DSM-IV de depressão maior ${ }^{9,10}$.
Nesse sentido, o Major Depression Inventory (MDI, Inventário de Depressão Maior) é um instrumento novo e foi desenvolvido com intuito de abranger o universo de sintomas do DSM-IV para depressão maior e CID-10 para depressão. Consiste de um questionário autoaplicável que contém os dez sintomas do CID-10 para depressão, que são idênticos aos sintomas do DSM-IV, com exceção da autoestima que, neste último, está incluída no sintoma culpa. Além disso, essa escala mede a gravidade dos estados depressivos.

O MDI já foi traduzido para o dinamarquês, holandês, inglês, finlandês, alemão, sueco e turco. A aplicabilidade e a validade do MDI foram examinadas em um número limitado de estudos e poucas traduções dessa escala foram publicadas ${ }^{11,12}$. A escala brasileira foi adaptada com base nas versões dinamarquesa, turca e holandesa do mesmo instrumento ${ }^{12}$.

Estudos da validação do $\mathrm{MDI}^{3,13,14}$ analisaram respectivamente a sensibilidade $(0,92 ; 0,66 ; 0,86)$ e a especificidade $(0,86 ; 0,63 ; 0,94)$ do MDI, estabelecendo pontos de corte de 26/27, relatando ser esse inventário confiável para a avaliação da depressão. Destacam ainda as características de simplicidade e rapidez de aplicação do MDI, a vantagem e a colaboração desse instrumento, em relação a outros existentes, pois permite aos clínicos avaliar a presença de um transtorno depressivo de acordo com o DSM-IV, e também a graduar a gravidade dos sintomas depressivos.

O MDI é classificado como um instrumento de rastreio curto $^{12}$, podendo ser aplicado em diferentes ambientes que avaliem a saúde mental.

Tendo em vista a grande utilidade da escala apresentada no rastreamento de sintomas depressivos e a importância das diferenças transculturais para aplicação em populações diferentes, torna-se essencial a padronização e validação do MDI para uso na população brasileira. Assim, será feita a utilização adequada do instrumento, auxiliando o diagnóstico da depressão e a criação de consensos para centros que desenvolvem atividades de pesquisa e assistência a pacientes com depressão.

O presente estudo teve por objetivos validar, adaptar e aferir a fidedignidade do MDI para a língua portuguesa

\section{MÉTODOS}

Primeiramente, obteve-se a autorização do autor do instrumento original para a tradução, adaptação e validação 13,15.

Em seguida, submeteu-se este trabalho ao Comitê de Ética da Universidade do Sul de Santa Catarina (Unisul), o qual foi aprovado sob o registro 08.160.4.01.III. Todos os participantes assinaram o Termo de Consentimento Livre e Esclarecido.

O MDI se refere a como o paciente tem se sentido nas duas últimas semanas. Contém dez itens, sendo que os itens 8 e 10 possuem dois subitens "a e b", considerando-se 
o subitem com a maior pontuação obtida. O MDI pode ser pontuado como uma escala Likert para depressão, podendo variar de 0 a 50. Cada item tem pontuação mínima de 0 (nenhuma vez) a 5 (o tempo todo), sendo que 20 pontos ou mais refere algum grau de depressão.

Essa escala pode ainda ser classificada dentro de um algoritmo, uma pontuação de 4 ou mais em um dos itens qualifica para o algoritmo da CID-10 ou DSM-IV. O algoritmo CID-10 classifica como depressão moderada uma pontuação de 4 ou 5 nos dois ou três primeiros itens somados a pelo menos quatro dos itens restantes. $\mathrm{O}$ algoritmo DSM-IV classifica como depressão maior uma pontuação de 4 ou 5 em cinco dos noves itens (item 4 ou 5 usa-se a maior pontuação); entre esses um deve ser humor deprimido ou falta de interesse?.

Quanto à forma de preenchimento, as escalas em geral podem ser de autoavaliação ou preenchidas por uma terceira pessoa ${ }^{5}$. O MDI tem como outro ponto positivo poder ser respondido tanto na forma autoaplicável quanto na forma de entrevista, sendo o tempo médio para preenchimento do MDI de 5 a 10 minutos.

O instrumento passou por dois tradutores independentes, ambos brasileiros, bilíngues, cientes do objetivo da pesquisa e especialistas em traduções na área da saúde. Foi dada ênfase à tradução conceitual e não à tradução literal de cada item. Após realizados os ajustes necessários pelos responsáveis pela pesquisa, obteve-se uma única versão (V1) do instrumento em português.

Essa V1 passou por duas retrotraduções realizadas por dois tradutores independentes, ambos brasileiros, bilíngues e sem conhecimento do objetivo da pesquisa. Após a compatibilização das duas versões, obteve-se uma única versão em inglês (V2). Foi realizada a comparação de V1 e V2 pelos pesquisadores e se realizaram os ajustes necessários, sendo constituída a versão 3 do questionário em português (V3).

Para a validação de face e conteúdo foi realizada uma revisão por um comitê de juízes. A versão V3 foi aplicada a seis profissionais da área da saúde, dentre eles quatro médicos psiquiatras especialistas em transtorno de humor e dois médicos generalistas. A esses profissionais foi dada breve orientação sobre equivalência semântica, idiomática, cultural e conceitual. Abaixo de cada item do MDI, constava a observação sobre a compreensão deste e, ao final da avaliação realizada por cada profissional, constava um espaço destinado a observações e sugestões.

A partir das observações e notas dos juízes, formulou-se, assim, a versão 4 (V4).

Na prova preliminar para realizar ajustes (pré-teste), a V4 foi aplicada aleatoriamente em 32 indivíduos que frequentavam a sala de espera de uma clínica de radiologia na cidade de Ponta Grossa (PR). Essa etapa teve como objetivos: confirmar a aplicabilidade do questionário, detectar eventuais dificuldades em sua aplicação, permitir a familiarização dos entrevistadores com o instrumento além de referendar a adaptação cultural.

Para testar a confiabilidade (teste-reteste), foram selecionados aleatoriamente 31 indivíduos alfabetizados, com ausência de transtornos mentais de acordo com psiquiatra da unidade pesquisada. Os sujeitos trabalhavam em um ambulatório de atendimento geral, aos quais o MDI foi aplicado duas vezes com intervalo de 1 a 2 semanas para avaliar a estabilidade do instrumento por meio da fidedignidade teste-reteste.

Para avaliação da consistência interna e validade concorrente, buscou-se a população atendida no Centro de Atendimento Psicossocial (CAPS) das cidades de Florianópolis (SC), Tubarão (SC) e Ponta Grossa, da qual foram selecionados os sujeitos que estavam presentes no período da coleta de dados. Foram excluídos os indivíduos que não possuíam nível mínimo de escolaridade (alfabetizados) para responder ao questionário, bem como os que possuíam concomitantemente o diagnóstico de outros distúrbios psiquiátricos, ficando a amostra composta por 30 pacientes com diagnóstico de depressão confirmado por médico psiquiatra por meio de entrevista clínica. Nesses pacientes, não foram controlados o tempo de tratamento, o uso de medicamentos e a gravidade do quadro depressivo. A esses foram aplicados o questionário sociodemográfico (idade, gênero e escolaridade), a versão em português do MDI (V4) e a escala de depressão de Hamilton (HAM-D) ${ }^{16}$. Por se tratar de unidades de saúde pública nas quais são atendidos indivíduos de baixa renda e escolaridade, houve dificuldade numa inclusão maior de indivíduos com as características solicitadas no estudo.

Em etapa posterior, esses pacientes foram comparados de modo 1:3 (um caso para três controles) por idade e sexo com um grupo controle de 90 indivíduos, selecionados de forma intencional nos CAPS, por meio dos prontuários médicos. Foram incluídos no grupo controle aqueles que não possuíam o diagnóstico de depressão ou outra doença psiquiátrica e com nível mínimo de escolaridade (saber ler) para responder ao questionário.

Dessa forma, a amostra foi composta conforme as etapas do estudo: construção do V1 e V2 por quatro tradutores; julgamento do V3 por seis profissionais especialistas; pré-teste por 32 sujeitos; teste-reteste por 31 indivíduos e aplicação da versão final em 30 pacientes com depressão e 90 controles, totalizando 193 pessoas.

Na análise estatística, foram realizados os procedimentos para consistência interna, utilizando-se o coeficiente alfa de Cronbach; para validade concorrente, o coeficiente de Pearson e a análise fatorial dos componentes principais, antes e após. A capacidade do instrumento em discriminar, em nossa população de estudo, as dimensões subjacentes à versão original da escala foi avaliada por meio da análise fatorial (o KMO - Kaiser-Meyer-Olkin; o índice de adequabilidade da amostra foi 0,500), aplicando-se o método dos eixos princi- 
pais (Principal Axis Factoring) para a extração de fatores ${ }^{17}$. Para avaliar o número de fatores a serem excluídos, utilizou-se o critério autovalores inferiores a 1; a rotação Varimax, em uma segunda fase, foi realizada para simplificar a interpretação dos fatores e minimizar o número de itens com grande peso em apenas um fator. A confiabilidade teste-reteste foi verificada por meio do Coeficiente de Correlação Intraclasse e pelo índice de correlação de Spearman. Também foi construída a curva Randomic Operator Characteristic (ROC), que permite evidenciar os valores para os quais existe maior otimização da sensibilidade em função da especificidade. Para tais análises, utilizou-se o pacote Statistical Package for the Social Sciences ${ }^{\circledR} 15.0$ e Stata $^{\circledR}$ 9.0.

\section{RESULTADOS}

O processo de validação, em seus estágios iniciais, em função das observações dos juízes, resultou em modificações em alguns itens do MDI no sentido de adaptá-lo à população brasileira. Assim, formulou-se a versão final V4.

A frase referente ao período de tempo "How much of the time..." foi traduzida para "Quanto do tempo...". Optou-se por agregar a essa tradução o período ao qual o questionário se refere de modo a facilitar a compreensão e enfatizar que esse instrumento avalia um período de duas semanas, originando assim a frase "Durante as duas últimas semanas".

Todas as questões obtiveram mais de 85\% de compreensão conforme sugerido por Guillemin et al.10; contudo, a questão 8B gerou alguma controvérsia entre os profissionais bem como entre os tradutores. A maior dificuldade encontrada nessa etapa foi em relação à palavra "subdued" (questão 8B) que, na V3, foi traduzida para a palavra "apagado", sendo substituída após a avaliação dos profissionais para a palavra "desanimado", que representava melhor culturalmente o objetivo da pergunta.

A questão 8B não foi referida por nenhum participante dessa etapa como incompreensível ou sem sentido, sendo mantida na forma mais próxima ao original: "Você se sentiu desanimado ou mais lento?".

As características sociodemográficas dos sujeitos da fase de aplicação do instrumento, grupo de casos $(n=30)$ e grupo controle $(n=90)$ estão descritas na tabela 1.

Tabela 1. Características sociodemográficas de casos com depressão e controles

\begin{tabular}{lcc}
\hline Características & Casos (depressão) & Controles \\
\hline Sexo: $\%(\mathrm{n})$ & Masculino: $30 \%(9)$ & Masculino: $30 \%(27)$ \\
& Feminino: $70 \%(21)$ & Feminino: $70 \%(63)$ \\
Idade: média (DP) anos & Média: $( \pm)$ 50,23 anos & Média: $( \pm)$ 50,16 anos \\
Escore MDI: média & Masculino: 32,22 & Masculino: 15,51 \\
& Feminino: 30,04 & Feminino: 11,57 \\
& Média (total): 30,70 & Média (total): 20,20 \\
\hline
\end{tabular}

DP: desvio-padrão; MDI: Inventário de Depressão Maior.
A consistência interna do MDI foi avaliada pelo coeficiente de fidedignidade de Cronbach, que foi igual a 0,917; este é um valor alto, sugerindo que o MDI reflete uma estrutura única. Os melhores índices de confiabilidade foram atingidos para os itens: $5(0,917), 8(0,914), 9(0,914), 10 \mathrm{~A}(0,914)$. A maioria deles apresentou índices muito bons de consistência interna, inclusive o item 10B, que apresentou a mais baixa correlação com todos os itens $(0,833)$.

A análise fatorial revelou a presença de dois fatores (Tabela 2). Os resultados antes e depois da rotação normalizada Varimax foram similares. O primeiro fator incluiu todos os itens, menos o 10B, que explica 53,9\% de variabilidade, sendo denominado nível geral de depressão. O segundo fator incluiu os itens 4, 5, 10B, que explicam 13,6\% de variabilidade. Juntos, os dois fatores explicam $67,6 \%$ da variabilidade.

Tabela 2. Fatores após análise fatorial referente aos itens do Inventário de Depressão Maior

\begin{tabular}{|c|c|c|c|c|c|}
\hline \multirow{2}{*}{ Item MDI } & \multirow{2}{*}{ Descrição do item } & \multicolumn{2}{|c|}{ Sem rotação } & \multicolumn{2}{|c|}{$\begin{array}{l}\text { Rotaçäo } \\
\text { Varimax }\end{array}$} \\
\hline & & Fator 1 & Fator 2 & Fator 1 & Fator 2 \\
\hline 1 & $\begin{array}{l}\text { Você se sentiu de baixo } \\
\text { astral ou triste? }\end{array}$ & 0,809 & 0,043 & 0,781 & 0,215 \\
\hline 2 & $\begin{array}{l}\text { Você perdeu interesse } \\
\text { em suas atividades } \\
\text { diárias? }\end{array}$ & 0,854 & $-0,087$ & 0,853 & 0,098 \\
\hline 3 & $\begin{array}{l}\text { Você sentiu falta de } \\
\text { energia ou força? }\end{array}$ & 0,884 & 0,155 & 0,830 & 0,340 \\
\hline 4 & $\begin{array}{l}\text { Vocêse sentiu menos } \\
\text { autoconfiante? }\end{array}$ & 0,856 & 0,243 & 0,784 & 0,421 \\
\hline 5 & $\begin{array}{l}\text { Você sentiu peso } \\
\text { na consciência ou } \\
\text { sentimento de culpa? }\end{array}$ & 0,554 & 0,391 & 0,457 & 0,500 \\
\hline 6 & $\begin{array}{l}\text { Você sentiu que viver } \\
\text { não vale a pena? }\end{array}$ & 0,881 & 0,018 & 0,857 & 0,206 \\
\hline 7 & $\begin{array}{l}\text { Você teve dificuldade } \\
\text { de concentração? Por } \\
\text { exemplo ao ler jornal } \\
\text { ou assistir TV? }\end{array}$ & 0,828 & $-0,126$ & 0,836 & 0,054 \\
\hline $8 \mathrm{~A}$ & Você se sentiu agitado? & 0,628 & $-0,081$ & 0,631 & 0,055 \\
\hline $8 B$ & $\begin{array}{l}\text { Você se sentiu } \\
\text { desanimado ou mais } \\
\text { lento? }\end{array}$ & 0,865 & 0,028 & 0,839 & 0,212 \\
\hline 9 & $\begin{array}{l}\text { Você teve problemas } \\
\text { para dormir à noite? }\end{array}$ & 0,655 & $-0,236$ & 0,691 & $-0,090$ \\
\hline $10 \mathrm{~A}$ & $\begin{array}{l}\text { Você esteve com } 0 \\
\text { apetite diminuído? }\end{array}$ & 0,679 & $-0,558$ & 0,782 & $-0,400$ \\
\hline \multirow[t]{3}{*}{ 10B } & $\begin{array}{l}\text { Você esteve com } 0 \\
\text { apetite aumentado? }\end{array}$ & 0,145 & 0,871 & $-0,045$ & 0,882 \\
\hline & Variância experimental & 6,72 & 1,3 & 6,47 & 1,63 \\
\hline & Proporção total (\%) & 56 & 11,6 & 53,9 & 13,6 \\
\hline
\end{tabular}

MDI: Inventário de Depressão Maior. 
A análise da consistência interna dos itens que compõem os fatores (precisão do fator), efetuada por meio do alfa de Cronbach, revelou que tais fatores apresentavam índices de fidedignidade iguais a 0,93 (fator 1) e 0,60 (fator 2).

O coeficiente de Pearson foi 0,56 ( $p<0,01)$ quando se compararam os valores totais do MDI com os valores da escala de Hamilton.

Em relação à avaliação da depressão grave, observou-se que o MDI acusou $56,6 \%(n=17)$ dos pacientes e a escala de depressão de Hamilton 53,3\% ( $n=16)$. Comparando as duas escalas, observa-se que o MDI identificou apenas um paciente a mais do que a escala de Hamilton.

Ao relacionarmos os valores do $\mathrm{MDI}$ com a presença e a ausência de depressão, obtivemos a curva ROC. O cálculo da sensibilidade e a especificidade na curva ROC demonstrou que a combinação ótima ocorreu com 0,86 para sensibilidade e 0,75 para especificidade no escore 16, com 30 casos e 90 controles corretamente classificados. A área abaixo da curva foi 0,88 . Isso quer dizer que um indivíduo com um escore igual ou superior a 16 é classificado como depressivo. A tabela 3 demonstra esses resultados.

Não houve diferença significativa nas médias entre o teste e o reteste. O coeficiente de correlação intraclasse para o total da escala foi 0,846 $(0,677-0,930)$ medidas simples e $0,917(0,807-0,964)$ medidas médias. A tabela 4 mostra o coeficiente de correlação intraclasse e o coeficiente de Spearman para cada item da escala.

O scatterplot bivariado entre os valores do teste e reteste do total do MDI (Figura 1) sugere que o total do MDI é uma variável confiável.

Tabela 3. Sensibilidade e especificidade do Inventário de Depressão Maior em vários pontos de corte

\begin{tabular}{cccc}
\hline Ponto de corte & Sensibilidade (\%) & Especificidade (\%) & $\begin{array}{c}\text { Corretamente } \\
\text { classificados (\%) }\end{array}$ \\
\hline 09 & 100,00 & 28,89 & 46,67 \\
10 & 96,67 & 36,67 & 51,67 \\
11 & 96,67 & 45,56 & 58,33 \\
12 & 93,33 & 48,89 & 60,00 \\
13 & 93,33 & 54,44 & 64,17 \\
14 & 90,00 & 60,00 & 67,50 \\
15 & 86,67 & 68,89 & 73,33 \\
16 & 86,67 & 75,56 & 78,33 \\
17 & 83,33 & 77,78 & 79,17 \\
18 & 80,00 & 80,00 & 80,00 \\
20 & 76,67 & 80,00 & 79,17 \\
21 & 73,33 & 85,56 & 82,50 \\
22 & 73,33 & 88,89 & 85,00 \\
23 & 70,00 & 91,11 & 85,83 \\
24 & 70,00 & 92,22 & 86,67 \\
\hline
\end{tabular}

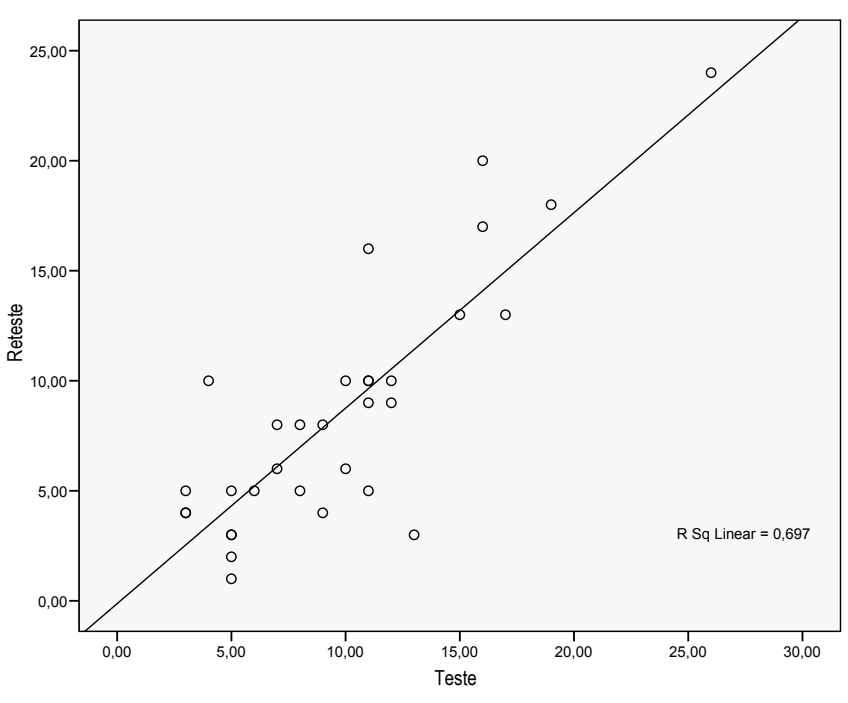

Figura 1. Scatterplot. R Sq linear: Linear Regression R-Squared.

Dos 30 pacientes com depressão (casos) diagnosticados por psiquiatras, 76,6\% (23) possuíam algum grau de depressão, usando o ponto de corte de 20 conforme sugerido pelo autor da escala. Utilizando o ponto de corte de 16, encontrado na análise da curva ROC nesse estudo, a proporção de pacientes deprimidos sobe para $86,6 \%$ (26).

O uso do algoritmo DSM-IV e CID-10 revelou uma performance razoável nessa população. A maioria dos pacientes com diagnóstico de depressão possuía mais de cinco critérios do DSM-IV (21 pacientes com mais de 5 versus 9 pacientes com menos de 5) e mais de 4 critérios do CID-10 (22 pacientes com mais de 4 versus 8 pacientes com menos de 4).

Para 30 pacientes, foi verificada a autoaplicabilidade do instrumento. A idade média dessa amostra foi de 50,23 anos $( \pm 7,27)$. Desses pacientes, $50 \%$ apresentavam baixa escolaridade (1० grau incompleto) e 50\% apresentavam maior escolaridade (1॰ grau completo). Para 46,6\% (14) da amostra, o questionário foi autoaplicável e 53,3\% (16) necessitaram de algum tipo de auxílio. Para os pacientes que realizaram de forma autoaplicável, a idade média foi de 42,28 \pm 7,05 anos, $64,28 \%$ (9) tinham maior escolaridade e $35,71 \%$ (5) tinham baixa escolaridade. Para os pacientes que necessitaram de algum tipo de auxílio, a idade média foi de 57,18 $\pm 11,17$ anos, $62,5 \%$ (10) possuíam baixa escolaridade e 37,5\% (6) possuíam maior escolaridade.

A escolaridade não foi fator estatisticamente significante ( $p>0,05)$ para a autorrealização do questionário. Entretanto, a idade foi fator que influenciou a autoaplicabilidade do questionário ( $p=0,01)$, visto que, os pacientes com idades acima de 60 anos (idosos), apresentaram limitações para responder sozinhos às questões $(p=0,024)$. 
Tabela 4. Confiabilidade teste-reteste

\begin{tabular}{|c|c|c|c|c|c|c|c|c|}
\hline \multirow{2}{*}{ Item MDI } & \multirow{2}{*}{ Descrição } & \multicolumn{2}{|c|}{ Teste } & \multicolumn{2}{|c|}{ Reteste } & \multirow{2}{*}{ ICC } & \multirow{2}{*}{ IC95\% } & \multirow{2}{*}{$r_{s}$} \\
\hline & & média & DP & média & DP & & & \\
\hline 1 & Você se sentiu de baixo astral ou triste? & 1,28 & 1,05 & 0,97 & 0,82 & 0,711 & $(0,41-0,86)$ & 0,48 \\
\hline 2 & Você perdeu interesse em suas atividades diárias? & 0,81 & 0,64 & 0,69 & 0,73 & 0,648 & $(0,28-0,83)$ & 0,54 \\
\hline 3 & Você sentiu falta de energia ou força? & 1,00 & 0,67 & 0,90 & 0,73 & 0,739 & $(0,46-0,87)$ & 0,49 \\
\hline 4 & Você se sentiu menos autoconfiante? & 0,93 & 1,07 & 0,78 & 0,83 & 0,786 & $(0,56-0,90)$ & 0,72 \\
\hline 5 & Você sentiu peso na consciência ou sentimento de culpa? & 0,87 & 0,83 & 0,69 & 0,78 & 0,501 & $(0,02-0,75)$ & 0,24 \\
\hline 6 & Você sentiu que viver não vale a pena? & 0,03 & 0,17 & 0,06 & 0,24 & 0,795 & $(0,58-0,90)$ & 0,70 \\
\hline 7 & Você teve dificuldade de concentração? Por exemplo ao ler jornal ou assistir TV? & 0,81 & 0,69 & 0,78 & 0,75 & 0,585 & $(0,15-0,79)$ & 0,44 \\
\hline $8 \mathrm{~A}$ & Você se sentiu agitado? & 1,43 & 1,29 & 1,12 & 0,83 & 0,734 & $(0,45-0,87)$ & 0,61 \\
\hline $8 B$ & Você se sentiu desanimado ou mais lento? & 1,00 & 0,80 & 0,87 & 0,87 & 0,816 & $(0,62-0,91)$ & 0,56 \\
\hline 9 & Você teve problemas para dormir à noite? & 1,03 & 1,33 & 0,59 & 1,07 & 0,930 & $(0,85-0,96)$ & 0,76 \\
\hline $10 \mathrm{~A}$ & Você esteve com o apetite diminuído? & 0,62 & 1,07 & 0,15 & 0,57 & 0,510 & $(0,01-0,76)$ & 0,41 \\
\hline 10B & Você esteve com 0 apetite aumentado? & 1,65 & 1,59 & 1,00 & 1,07 & 0,832 & $(0,66-0,92)$ & 0,66 \\
\hline MDI escore total & & 9,78 & 5,25 & 8,56 & 5,58 & 0,909 & $(0,81-0,96)$ & 0,73 \\
\hline
\end{tabular}

MDI: Inventário de Depressão Maior; DP: desvio-padrão; ICC: índice de correlação intraclasse; IC95\%: intervalo de confiança de 95\%; r: índice de correlação de Spearman.

\section{DISCUSSÃO}

A decisão da escolha de um instrumento de autoavaliação ou heteroavaliação deve levar em consideração vários aspectos, como nível educacional, disponibilidade de tempo do entrevistador e objetivo da avaliação.

Escalas de autoavaliação, como MDI, são dependentes da cooperação do paciente e da habilidade de "ler" deste; por outro lado, essas escalas poupam tempo do examinador.

Com o objetivo de auxiliar no diagnóstico e no acompanhamento da evolução da doença depressiva, o presente estudo oferece dados preliminares para a obtenção das propriedades da versão em português do MDI. Isso faz com que mais estudos sejam necessários sobre essa escala em um número maior de indivíduos.

A consistência do MDI medida pelo alfa de Cronbach foi boa quando tomada todas as questões, todos os fatores ou o fator 1 isoladamente, porém não é boa quando se toma o fator 2 isoladamente.

Bech e Wermuch ${ }^{15}$ e Bech et al..$^{13}$, utilizando o MDI em diferentes estudos, encontraram alfas de Cronbach de 0,92 e 0,94. Esses valores são comparáveis com 0,91 encontrado no presente estudo. Valores acima de 0,80 traduzem uma consistência interna adequada ${ }^{18}$. A validação do MDI na população grega ${ }^{7}$ encontrou um alfa de Cronbach para a escala total de 0,89, e a análise fatorial revelou resultados semelhantes. No nosso estudo, observou-se que o índice alfa de Cronbach aumentaria para 0,933 se a questão 10 fosse transformada em um único item, em vez da subdivisão em "a e b", o poderia facilitar a compreensão da questão.

A validade de critério foi analisada pela medida das correlações entre o escore final do MDI e da Escala de Hamilton.
Observou-se que houve, de maneira geral, uma fraca associação entre os dois instrumentos. Isso pode ter ocorrido pelo fato de que o MDI é baseado no universo do DSM-IV e CID-10, enquanto a escala de Hamilton foi criada fora desse contexto.

Por outro lado, Olsen et al. ${ }^{19}$, avaliando as propriedades psicométricas do MDI em população dinamarquesa, encontraram um coeficiente de Pearson de 0,86 entre essas duas escalas. Portanto, futuros estudos comparando outras escalas de depressão, como a de Beck ${ }^{9}$ ou de Zung ${ }^{20}$, ou até mesmo a de Hamilton em uma população maior, poderão mostrar uma melhor associação entre essas escalas. Quando analisamos depressão grave, essas escalas apresentaram desempenho similar.

O conhecimento de uma escala e seu melhor ponto de corte é importante para o gerenciamento desse material disponível nos espaços públicos de atenção à saúde mental. Diferentes estudos com o MDI, ${ }^{7,1,14}$ encontraram o ponto de corte de 26/27. Em nosso estudo, utilizando a curva ROC, encontramos um ponto de corte de 16 , com sensibilidade de 0,86 e especificidade de 0,75 . Utilizando o ponto de corte do nosso estudo, conseguimos englobar mais casos de depressão entre os pacientes que tinham o diagnóstico prévio dessa patologia.

O estudo de Forsell7, que analisou a associação entre o MDI e o instrumento padronizado de avaliação clínica em neuropsiquiatria (SCAN) em base populacional, numa amostra de 1.093 indivíduos, cita que a melhor pontuação de corte do MDI deve ser escolhida de acordo com os objetivos do estudo. Optamos, assim, por uma maior sensibilidade, visto que as escalas de avaliação de depressão visam primordialmente ao rastreamento e, secundariamente, ao diagnóstico. 
Estudo de revisão de vários instrumentos autoaplicáveis para detecção da depressão sugere que não há diferença significativa entre eles em termos de performance. Em todos os instrumentos, a sensibilidade está em torno de 0,84 e a especificidade, em torno de $0,72^{16}$, corroborando os resultados encontrados neste estudo.

O uso dos algoritmos DSM-IV e CID-10 encontram respaldo nos resultados desse estudo, apresentando um desempenho razoável, visto que a maioria dos pacientes com depressão preenchia muitos dos critérios necessários para esses algoritmos. Portanto, recomendamos sua utilização. Diferentemente, a validação grega do MDI não encontrou um bom desempenho desses algoritmos e não recomenda sua utilização na população daquele estudo .

Em relação à fidedignidade teste-reteste, não houve diferença entre as médias da primeira medida (teste) quando comparada com a segunda (reteste). Os coeficientes de correlação das medidas foram significativos com valores superiores a 0,5, o que demonstra a estabilidade do instrumento.

A escala teve boa aceitação pelos participantes dessa pesquisa. Quanto à autoaplicabilidade do questionário, observou-se que os pacientes idosos (acima de 60 anos) apresentaram maior dificuldade. Os autores desse estudo sugerem que, para essa faixa etária, o instrumento MDI seja aplicado em forma de entrevista. Outros estudos, em população europeia com indivíduos entre 18 e 79 anos envolvendo o MDI, referem que nenhum participante teve algum problema em entender as questões ${ }^{7,21}$. Mais estudos são necessários com pacientes idosos.

Uma das limitações do presente estudo esteve relacionada ao fato de muitos dos pacientes depressivos estarem em tratamento. $\mathrm{O}$ uso de medicação foi um fator que pode ter influenciado na resposta do MDI nesses pacientes. A retirada da medicação ou postergação do início do tratamento não foi solicitada pelos responsáveis pela pesquisa por questões éticas. Outro fator que pode ter influenciado o baixo ponto de corte foi o não controle da gravidade do quadro depressivo, assim como o tempo de tratamento e a medicação utilizada.

O resultado desse estudo revela que o MDI demonstra ser apropriado para o uso na população brasileira, apresentando alta sensibilidade e especificidade no ponto de corte 16, alta confiabilidade teste-reteste e alta consistência interna.

\section{CONCLUSÃO}

A versão do MDI traduzida e validada para o português, de acordo com o resultado da análise final de suas propriedades psicométricas, demonstra ser um instrumento adequado que permite avaliar a presença de um transtorno depressivo de acordo com o DSM-IV e também a gravidade dos sintomas depressivos.

\section{REFERÊNCIAS}

1. Fountoulakis K, Apostolos I, Kleanthous S, Samolis S, Kaprinis SG, Sitzoglou K, et al. Reliability, validity and psychometric properties of the Greek translation of the Center Epidemiological Studies-Depression (CES-D) Scale. BMC Psychiatry. 2001;1:3.

2. Dractu L, Ribeiro LC, Calil HM. Escalas de Avaliação da Depressão e sua utilidade clínica: Hamilton, Montgomery. Åsberg e Visual Análoga do Humor. Rev Psiquiatr. 1985;7:59-65.

3. Cuijpers P, Dekker J, Noteboom A, Smits N , Peen J. Sensibilidade e especificidade do Inventário de Depressão Maior em nível ambulatorial. BMC Psychiatry. 2007;7:39.

4. Moreno R, Moreno D. Escalas de Depressão de Montgomery e Asberg (MADRS) e de Hamilton (HAM-D). Rev Psiq Clínica. 1998;25(5):50-63.

5. Kaplan HI, Sadock BJ, Greeb JA. Compêndio de psiquiatria: ciências do comportamento e psiquiatria clínica. 7. ed. Porto Alegre: Artmed; 1997.

6. Calil HM, Pires MN. Aspectos gerais das escalas de avaliação de depressão. Rev Psiq Clin. 1998;25:43-56.

7. Forsell Y. The Major Depression Inventory versus Schedules for Clinical Assessment in Neuropsychiatry in a population sample. Soc Psychiatry Psychiatr Epidemiol. 2005;40(3):209-13.

8. Giovani A, Melo GF, Parente I, Dantas G. Elaboração e validação da Escala de Depressão para Idosos. Cad Saúde Pública. 2008;24:975-82.

9. Beck A, Ward C, Mendelson M, Mock J, Erbaugh J. An inventory for measuring depression. Arch Gen Psychiatry. 1961;:453-63.

10. Guillemin F, Bombardier C, Beaton D. Cross-cultural adaptation of health-related quality of life measures. J Clin Epidemol. 1993;46(12):1417-32.

11. Evidência clínica: conciso. 11. ed. Porto Alegre: Artmed; 2005

12. Mulrow C, Williams J, Gerety M, Ramirez G, Montiel 0, Kerber C. Case-finding instruments for depression in primary care settings. Ann Intern Med. 1995;122(12):913-21.

13. Bech P, Rasmussen N, Olsen LR, Noerholm V, Albidgaar W. The sensitivity and specificity of the MDI using the present State Examination as the index of diagnostic validity. J Affect Disord. 2001;66(2-3):159-64.

14. Fountoulakis KN, lacovides A, Kleanthous S, Samolis S, Gougoulias K, Tsiptsios I, et al. Reliability, validity and psychometric propreties of the Greek translation of the Major Depression Inventory. BMC Psychiatric. 2003;3:2.

15. Bech P, Wermuch L. Applicability and Validity of the MDI in patients with Parkinson's Disease. Nord J Psychiatr. 1998;52:305-9.

16. Menezes PR. Validade e confiabilidade das escalas de avaliação em psiquiatria. Rev Psiq Clin. 1998;25(5):26-37.

17. Norman GR, Streiner DL. Biostatistics: the bare essentials. 2. ed. London: BC Decker; 2000.

18. Olsen R, Jensen DV, Noerholm M, Martiny K, Bech P. The internal and external validity of the Major Depression Inventory in measuring severity of depressive states. Psychol Med. 2003;33(2):351-6

19. Olsen LR, Mortensen EL, Bech P. Prevalence of major depression and stress indicators in the Danish general population. Acta Psychiatr Scand. 2004;109(2):96-103.

20. Zung WWK. A self-rating depression scale. Arch Gen Psychiatry. 1965;12:63-70.

21. Organização Mundial da Saúde (OMS). Classificação estatística internacional de doenças e problemas relacionados à saúde CID-10. 5. ed. São Paulo: EDUSP; 1997. 